\title{
Imaging Center - Optimierung der diagnostischen Bildgebung im DRG-Zeitalter
}

\author{
Imaging Center - Optimization of the Imaging Process
}

Autor

Institut
H.-P. Busch

Abteilung für Radiologie, Neuroradiologie, Sonographie und Nuklearmedizin, Krankenhaus der Barmherzigen Brüder
Key words

- health policy and practice

- imaging sequences

- economics

- imaging pathway

- imaging center

eingereicht 3.7.2012

akzeptiert $\quad 7.11 .2012$

Bibliografie

DOI http://dx.doi.org/

10.1055/s-0032-1330346

Online-Publikation: 11.1.2013

Fortschr Röntgenstr 2013; 185:

313-319 @ Georg Thieme

Verlag KG Stuttgart · New York .

ISSN 1438-9029

Korrespondenzadresse

Prof. Hans-Peter Busch

Abteilung für Radiologie, Neuroradiologie, Sonographie und Nuklearmedizin,

Krankenhaus der Barmherzigen Brüder

Nordallee 1

54292 Trier

Tel.: ++49/651/2082840

Fax: ++49/6 51/2082842

h-p.busch@bk-trier.de

\section{Zusammenfassung \\ $\nabla$}

Weltweit stehen Krankenhäuser unter wachsendem Druck, die Wirtschaftlichkeit von Behandlungsprozessen zu optimieren, d. h. zu verbessern. Dabei hat die bildgebende Diagnostik einen großen Anteil am klinischen Erfolg, aber auch an den Kosten der Behandlung. Im klinischen Alltag fördert ein Überangebot bildgebender Methoden häufig eine „Sowohl-als-auch“-Strategie bis zur Kapazitätsgrenze ohne kritische Reflektion. Leistungen, die auf das klinische Gesamtergebnis des Patienten (clinical outcome) vorhersehbar keinen Einfluss haben, stellen eine nicht gerechtfertigte Belastung für den Patienten dar. Sie sind aus ökonomischer Sicht sinnlos und damit für die Existenz des Krankenhauses bedrohlich. Während bisher die Ziele der Optimierungsstrategie fast ausschließlich auf Qualität und Effizienz der Durchführung einzelner Untersuchungen gerichtet waren, muss in Zukunft die Effektivität einzelner Methoden im Gesamtprozess bewertet werden. Überflüssige Leistungen können nur vermieden werden, wenn die Optimierung des gesamten Imaging-Prozesses (Effizienz und Effektivität) im Vordergrund steht. Dies erfordert eine neue Definition der Prozesse (Imaging Pathway), neue organisatorische Strukturen (Imaging Center) und eine neue Denkweise der am Behandlungsprozess beteiligten Personen. Das Anreiz- und Motivationssystem muss sich wandeln von der Belohnung der gemachten Leistungsmenge zur Belohnung der Prozessqualität (medizinische Qualität, Servicequalität, Wirtschaftlichkeit) und damit auch der vermiedenen (nicht notwendigen) Leistungsmenge.

Die Rahmenbedingungen im Gesundheitssystem verlangen von Krankenhäusern Behandlungsabläufe mit hoher medizinischer Qualität und Wirtschaftlichkeit. Im Wettbewerb der Leistungsanbieter ergibt sich daraus ein Zwang zur

\section{Abstract \\ $\nabla$}

Hospitals around the world are under increasing pressure to optimize the economic efficiency of treatment processes. Imaging is responsible for a great part of the success but also of the costs of treatment. In routine work an excessive supply of imaging methods leads to an "as well as" strategy up to the limit of the capacity without critical reflection. Exams that have no predictable influence on the clinical outcome are an unjustified burden for the patient. They are useless and threaten the financial situation and existence of the hospital. In recent years the focus of process optimization was exclusively on the quality and efficiency of performed single examinations. In the future critical discussion of the effectiveness of single exams in relation to the clinical outcome will be more important. Unnecessary exams can be avoided, only if in addition to the optimization of single exams (efficiency) there is an optimization strategy for the total imaging process (efficiency and effectiveness). This requires a new definition of processes (Imaging Pathway), new structures for organization (Imaging Center) and a new kind of thinking on the part of the medical staff. Motivation has to be changed from gratification of performed exams to gratification of process quality (medical quality, service quality, economics), including the avoidance of additional (unnecessary) exams.

kontinuierlichen Optimierung der Gesamtbehandlungsprozesse von der Aufnahme über teilweise mehrere Abteilungen bis zur Entlassung. Im System der Fallpauschalen wird für das Krankenhaus ein abteilungsübergreifendes, interdis- 
ziplinäres und interprofessionelles Fallmanagement überlebensnotwendig. Erfolgsparameter sind das medizinische Endergebnis, die Patientenzufriedenheit und, ebenso wichtig für das Überleben des Krankenhauses, die Wirtschaftlichkeit im Erlössystem der Fallpauschalen (DRG) $[1,2,13,16]$.

Neben den strukturellen und organisatorischen Voraussetzungen ist für eine erfolgreiche Umsetzung dieser Optimierungsstrategie das Bewusstsein der Mitarbeiter ausschlaggebend, in Gesamtprozessen zu denken, zu handeln und zu optimieren [14, 15]. Dies stellt einen Paradigmenwechsel dar: von der Betrachtung der eigenen „Fachinsel“ hin zur Optimierung der eigenen Leistungen im Kontext von Gesamtprozessen. Am Ende zählt in qualitativer und wirtschaftlicher Hinsicht nur das Gesamtergebnis. Der Wettbewerb ist ein Qualitäts- und Kostenwettbewerb von Gesamtprozessen [3].

Daher gilt:

1. Die Rahmenbedingungen im Gesundheitssystem geben als Kriterien für die Optimierung von Gesamtbehandlungsprozessen im Krankenhaus Qualität und Wirtschaftlichkeit vor.

2. Das Krankenhaus muss diese Kriterien akzeptieren und (neue) Aufbau- und Ablaufstrukturen für eine systematische Prozessoptimierung schaffen.

3. Geeignete Führungskräfte müssen für die neuen Strukturen und Prozesse gefunden und qualifiziert werden (erst die notwendigen Prozesse definieren - dann geeignete Strukturen schaffen dann qualifizierte und motivierte Personen finden/binden/entwickeln).

In den Behandlungsabläufen im Krankenhaus stellt die diagnostische Bildgebung einen bedeutenden Qualitäts-, aber auch Kostenfaktor dar. Für die moderne Radiologie ergibt sich aus den genannten Rahmenbedingungen zwingend die Herausforderung, bei jedem Patienten nicht nur einzelne angeforderte Untersuchungen abzuarbeiten, sondern den gesamten Imaging-Prozess in den Blick zu nehmen und unter dem Aspekt der medizinischen Qualität und der Kosten zu optimieren [4]. Im Überangebot diagnostischer Möglichkeiten muss der Radiologe die Aufgabe des Lotsen auf dem Imaging Pathway übernehmen. Kriterien für eine Prozessoptimierung sind dabei das diagnostische Gesamtergebnis, die Servicequalität, die Effizienz der Durchführung und die Effektivität der einzelnen Untersuchungen. Dies erfordert ein radikales Umdenken bei den Zuweisern, aber auch bei den Radiologen.

Während bei einer Praxis der Fokus auf der effizienten Erbringung von Einzelleistungen liegt, muss der Fokus eines Imaging Centers auf effizienten und effektiven Imaging Pathways liegen. Dabei stellt insbesondere im DRG-Zeitalter die interdisziplinäre Beratung vor der Diagnostik von Krankheitsbildern verbunden mit Vorschlägen zur medizinisch und ökonomisch effizienten und effektiven Gestaltung von Imaging Pathways eine spannende Herausforderung für die Radiologie dar. Eine durch festgelegte Behandlungspfade oder interdisziplinäre Gespräche vermiedene CT-Untersuchung ist sowohl für den Patienten als auch für die Wertschöpfungskette des Krankenhauses ein Vorteil. Der Nachteil aus der „Inselsicht“ Radiologie (weniger Leistungen = weniger Personal $=$ Verkleinerung der Abteilung) sollte der Vergangenheit angehören. Personalinvestitionen in eine Steigerung der Prozessqualität stellen im Wettbewerb von Qualität und Gesamtkosten einen Vorteil für Patienten und Krankenhäuser dar. Das moderne Imaging Center muss im Gesundheitsmarkt ständig neue Anwendungen (z.B. virtuelle Koloskopie, Kardio-CT, Ganzkörper-MRT, Spektroskopie) eröffnen und auf den Prüfstand der medizinischen und ökonomischen Verbesserung von Behandlungsabläufen stellen („Innovation statt Reduktion“).

\section{Imaging Prozesse - von der Summe der Einzel- untersuchungen zum optimierten Imaging-Pfad im DRG-System}

Die Wertschöpfung einer DRG wird durch das Verhältnis von Gesamtkosten zum DRG-Erlös (Fallpauschale) dargestellt. Die Gesamtkosten für die Leistungserbringung setzen sich zusammen aus der Art und Anzahl der Leistungen und den Kosten pro Leistung. In den Fallpauschalen können den zugehörigen Personalund Materialkosten sowie den Kosten für die Infrastruktur Anteile der kalkulierten Fallpauschale zugeordnet werden. Dabei werden separat Fallpauschalen Anteile mit einer Aufstellung der im Mittel durchgeführten Untersuchungen für die Bildgebung ausgewiesen (INEK Tabelle Spalte 9) (www.g-drg.de) [5]. Für die Radiologie müssen gegebenenfalls bei einzelnen DRGs Diagnostik/Therapieund Strahlentherapie-Anteile, aber auch Anteile der Sonografie separiert werden.

In Zukunft wird mit steigender Tendenz eine ökonomische Beurteilung der Personal- und Sachkosten in der Radiologie an den Imaging-Pauschalen „Radiologie“ der DRG-Kalkulation erfolgen. Das Ergebnis muss wegen der vorgegebenen Kalkulationsbasis und der Frage der Vergleichbarkeit der Krankenhäuser mit großer Sorgfalt beurteilt werden, kann aber Hinweise auf die Notwendigkeit zur Optimierung von Imaging-Prozessen geben. Ein Benchmarking an DRG-Anteilen darf eine Abteilung (einen Chefarzt) nicht überraschen und unvorbereitet treffen! Dabei sollte das Benchmarking an DRG-Anteilen von den Abteilungen/Zentren nicht als Bedrohungsszenarium, sondern als Ausgangspunkt für eine erfolgreiche Prozessoptimierung gesehen werden [6]. Grundvoraussetzung für die kritische Auseinandersetzung mit dem Thema Benchmarking sind ausreichende Methodenkenntnis bei den Führungskräften einer Abteilung/eines Zentrums und ein Verständnis der eigenen Daten und der berechneten Ergebnisse. Dies bedarf einer systematischen Personalentwicklung und der Schaffung von Strukturen und Werkzeugen.

Die Optimierung der gesamten Bildgebung eines Patienten erfordert bei der Leistungssteuerung neben der medizinischen Qualitätskontrolle ein Monitoring der Effizienz (Wurde die MRT-Untersuchung kostengünstig erbracht?) und der Effektivität (War die CT-Untersuchung notwendig? Gab es eine kostengünstigere diagnostische Alternative mit gleicher medizinischer Information?). Dies ist nicht Aufgabe der Verwaltung, sondern der Abteilungsbzw. Zentrumsleitung (internes Bereichscontrolling) ( $\bullet$ Abb. 1). Gemeinsames Ziel der Prozessoptimierung in Praxis und Klinik ist die kostengünstige Erbringung von Einzelleistungen (Effizienz) mit hohem Qualitätsanspruch. Auch die Radiologie im Krankenhaus muss sich dabei der Forderung nach einer konti-

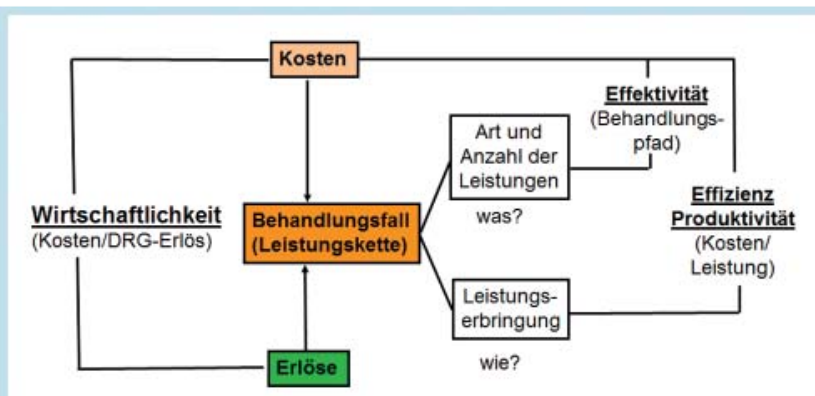

Abb.1 Wirtschaftlichkeit von Behandlungsprozessen im DRG-System 
nuierlichen Steigerung der Produktivität stellen. Dies bedeutet ein Monitoring und eine daraus resultierende Steuerung der Auslastung einzelner Untersuchungsgeräte (Utilization Management) und der personellen und materiellen Ressourcenzuteilung. Eine von der Klinikleitung beauftragte Analyse der Produktivität durch einen externen Unternehmensberater darf den Chefarzt nicht unvorbereitet treffen!

Die Optimierung der Effektivität einzelner diagnostischer Leistungen an Leitlinien und Imaging Pathways unterscheidet die Krankenhausradiologie von einer radiologischen Praxis. Dabei stellen standardisierte, evidenzbasierte und kostensensitive Behandlungsprozesse (Imaging Pathways) die Voraussetzung für eine erfolgreiche Optimierungsstrategie in den Rahmenbedingungen der DRGWelt dar. Sie machen Prozesse definier- und messbar - und damit optimierbar. Davon profitieren Patienten und Krankenhaus. Beschriebene Behandlungspfade ohne Monitoring sind zwar nützlich für Zertifizierungen, jedoch nicht geeignet für die kontinuierliche Verbesserung täglicher Behandlungsabläufe. Für den Teilprozess Imaging erlaubt ein leistungsfähiges RIS mit Vernetzung zum KIS das kontinuierliche Monitoring der Imaging-Pfade.

Im Krankenhaus findet in der Regel keine systematische Analyse der Behandlungsabläufe in den Dimensionen medizinische Qualität, Servicequalität und Wirtschaftlichkeit statt - und damit wird Optimierungspotenzial verschenkt. Retrospektive Analysen einzelner Behandlungsabläufe werden in der Regel nur im Rahmen der medizinischen Qualitätssicherung oder bei Schadensfällen durchgeführt. Auch bei hervorragendem klinischen Outcome für den Patienten und guter Verschlüsselung kann der Behandlungsweg zahlreiche nicht notwendige Untersuchungen, Behandlungsschritte und Pflegemaßnahmen enthalten, die im einfachsten Fall „nur“ ökonomisch sinnlos sind, häufig aber auch eine zusätzliche Belastung und Gefährdung des Patienten darstellen. Die kritische retrospektive Analyse, welche Untersuchungen einem verschwendeten Ressourcenverbrauch und einer nicht notwendigen Belastung des Patienten entsprechen, ist für Radiologen, technische Assistenten, aber auch für das zentrale Controlling und das zentrale Qualitätsmanagement noch eine ungewohnte Herausforderung. Aus dieser Analyse müssen dann prospektive Konsequenzen für zukünftige Abläufe gezogen werden.

Für die diagnostische Bildgebung im DRG-Zeitalter sollten angestoßen von Monitoring-Programmen oder individuellen Erfahrungen monatliche Fallkonferenzen zusammen mit den klinischen Partnern zur Analyse von nicht optimalen Imaging-Prozessen abgehalten werden. Neben einer Verbesserung zukünftiger Abläufe steigern die Konferenzen bei Klinikern und Radiologen die Sensibilität für Ressourcenverschwendung und unnötige Belastung des Patienten.

\section{Das Imaging Center - eine neue Organisationsform für die diagnostische Bildgebung im DRG-System}

$\nabla$

Die Optimierung von Gesamtbehandlungsprozessen (Imaging) erfordert eine Gesamtorganisation/Gesamtstruktur - das „Imaging Center“. Die Schaffung eines „Imaging Centers“ muss strategisches Ziel der Krankenhausleitung und der radiologischen Abteilungs-/ Zentrumsleitung sein. Dazu müssen die notwendigen organisatorischen Voraussetzungen (EDV, Stabsstellen) geschaffen und geeignete und motivierte Führungskräfte und Mitarbeiter gefunden und entwickelt werden. Die Idee von Imaging Pathways, die in (von) einem Imaging Center gesteuert werden, muss auch von klinischen Abteilungen und Kollegen als Erfolgsmodell anerkannt sein (Win-

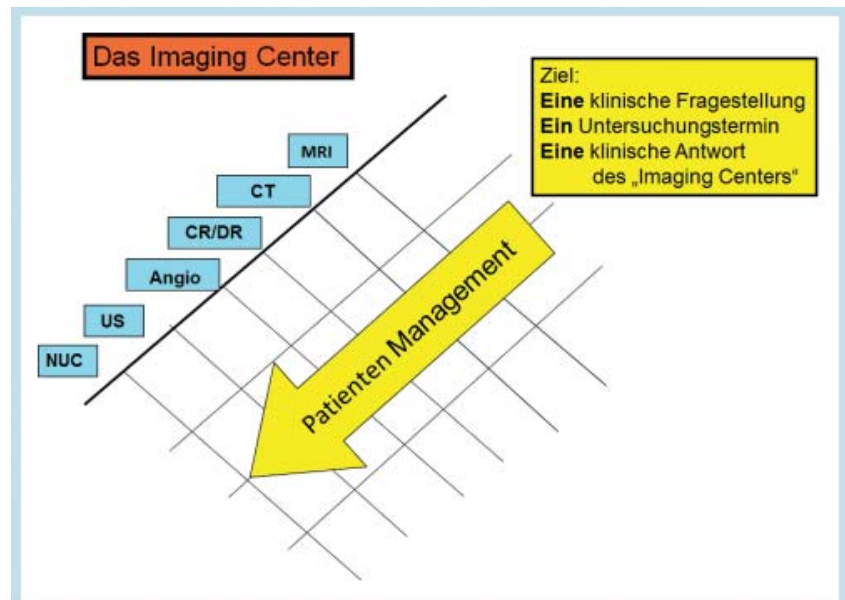

Abb. 2 Patientenmanagement im Imaging Center.

win-Situation). Hier hat insbesondere der radiologische Chefarzt eine schwierige persönliche Überzeugungsarbeit bei allen Beteiligten zu leisten. Nur wenn diese Vorbedingungen erfüllt sind, kann erfolgreich ein Imaging Center eingeführt werden ( $\bullet$ Abb. 2).

Die Verantwortung für einen gesamten Imaging Pathway erfordert andere organisatorische und damit strukturelle Voraussetzungen als die Durchführung zahlreicher Einzelleistungen. Gesamtverantwortung kann nur in einem Imaging Center übernommen werden, in dem sämtliche Methoden der Bildgebung (Röntgen, CT, MRT, Nuklearmedizin, Sonografie) auf hohem medizinischem Niveau integriert sind. Aufgabe der Klinikleitung ist hierbei häufig eine schwierige Umverteilung von Besitzständen (Beispiel: zentrale Sonografie). Die Steuerung und Optimierung von Gesamtprozessen erfordert Zeit und Können, d.h. Personalstellen für das Monitoring und die Optimierung der Prozessqualität im Zentrum. Diese zusätzlichen Stellen werden sich nicht direkt als zusätzliche Leistungserbringung für das Zentrum rechnen (Kennzahlen z. B. Anzahl der CT-Untersuchungen pro Facharzt), sondern über eine Optimierung (Minimierung) des notwendigen Ressourcenverbrauchs für den Gesamtprozess. In diesem Fall wird in die Steigerung der Prozessqualität investiert - ein Vorteil für den Patienten und die Ökonomie des Krankenhauses. Das Imaging Center ist keine Praxis im Krankenhaus!

Die Optimierung der Gesamtprozesse erfordert neben einem Umdenken der Beteiligten (systematische Personalentwicklung) ein erweitertes Kennzahlensystem. Benötigt werden wie bisher Kennzahlen für die Produktivität, jetzt aber auch Kennzahlen für die Effektivität, z.B. radiologisches Leistungsvolumen und Leistungsspektrum pro DRG im Vergleich zum Vorjahr, zur DRG-Kalkulation oder zu vergleichbaren externen Wettbewerbern. Für diese Kennzahlen ist die Verknüpfung der DRG-Daten des KIS mit der Leistungserfassung im RIS notwendig. Die Festlegung einer „ArbeitsDRG“ am Anfang der Diagnostikkette startet mit einer Benachrichtigung an die Radiologie automatisch das kontinuierliche Monitoring des entsprechenden Imaging Pathways. Das KIS liefert für jeden Patientenaufenthalt die verschlüsselte Fallpauschale, das RIS die Art, den Zeitpunkt und den Auftraggeber der erbrachten Leistungen. Ist der interne Punktwert für die Personalkosten und die Zuordnung der Materialkosten bekannt, können entsprechend der DRG-Kalkulation die Personal- und Sachkosten im Einzelfall oder als Mittelwert mit dem Anteil an der entsprechenden Fallpauschale 


\begin{tabular}{|c|c|c|c|c|c|c|c|c|}
\hline $\begin{array}{l}\text { DRG- } \\
\text { VERSION }\end{array}$ & DRG & LEISTUNGSBEZ & FALLNUMMER & Inek gesamt & $\begin{array}{l}\text { IST Personal } \\
\text { +Material }\end{array}$ & $\begin{array}{l}\text { Soll Personal } \\
\text { +Material DRG }\end{array}$ & $\begin{array}{l}\text { Diff SOLL/IST } \\
\text { DRG }\end{array}$ & $\begin{array}{l}\text { Relation } \\
\text { SOLL/IST }\end{array}$ \\
\hline \multirow{17}{*}{ GDRG_2011 } & \multirow{17}{*}{ B70D } & \multirow{17}{*}{$\begin{array}{l}\text { Apoplexie ohne } \\
\text { komplexen } \\
\text { zerebrovask. } \\
\text { Vasospasmus, } \\
\text { ohne } \\
\text { komplizierende } \\
\text { Diagnose oder } \\
\text { systemische } \\
\text { Thrombolyse, } \\
\text { mit neurol. } \\
\text { Komplexbeh. } \\
\text { des akuten } \\
\text { Schlaganfalls } \\
\text { bis } 72 \text { Std. oder } \\
\text { mit anderer } \\
\text { neurol. } \\
\text { Komplexbeh. } \\
\text { des akuten... }\end{array}$} & $2: 0350$ & $3.789,59$ & 286,35 & 323,55 & 37,20 & 1,13 \\
\hline & & & & $3.789,59$ & 364,24 & 323,55 & $-40,68$ & 0,89 \\
\hline & & & 339760 & $3.789,59$ & 291,63 & 323,55 & 31,92 & 1,11 \\
\hline & & & 30001 & $3.789,59$ & 315,32 & 323,55 & 8,23 & 1,03 \\
\hline & & & & $3.789,59$ & 90,66 & 323,55 & 232,89 & 3.57 \\
\hline & & & 3391502 & $3.789,59$ & 294,57 & 323,55 & 28,99 & 1,10 \\
\hline & & & 339942 & $3.789,59$ & 208,89 & 323,55 & 114,66 & 1.55 \\
\hline & & & 339 & $3.789,59$ & 466,62 & 323,55 & $-143,07$ & 0,69 \\
\hline & & & 0.005 & $3.789,59$ & 526,86 & 323,55 & $-203,31$ & 0,61 \\
\hline & & & $-9+213$ & $3.789,59$ & 258,18 & 323,55 & 65,37 & 1.25 \\
\hline & & & $339 \sqrt{7}$ & $3.789,59$ & 85,96 & 323,55 & 237,59 & 3.76 \\
\hline & & & 3397 & $3.789,59$ & 98,29 & 323,55 & 225,27 & 3.29 \\
\hline & & & & $3.789,59$ & 222,94 & 323,55 & 100,61 & 1.45 \\
\hline & & & 23 & $3.789,59$ & 372,31 & 323,55 & $-48,76$ & 0,87 \\
\hline & & & & $3.789,59$ & 90,66 & 323,55 & 232,89 & 3.57 \\
\hline & & &  & $3.789,59$ & 109,14 & 323,55 & 214,41 & 2.96 \\
\hline & & & & $3.789,59$ & 281,66 & 323,55 & 41,90 & 1,15 \\
\hline
\end{tabular}

Abb. 3 Vergleich der Personal- und Sachkosten des Imaging Centers im Krankenhaus der Barmherzigen Brüder Trier mit den kalkulierten DRG-Anteilen.

oder bei einem Benchmarking mit externen Leistungsanbietern verglichen werden.

- Abb. 3 zeigt einen Vergleich der Personal- und Sachkosten des Imaging Centers im Krankenhaus der Barmherzigen Brüder Trier mit den kalkulierten DRG-Anteilen bei einzelnen Patienten der DRG B70D. In einer retrospektiven Analyse aus medizinischer und ökonomischer Sicht der bildgebenden Verfahren bei der Akutbehandlung des ischämischen Schlaganfalls konnte gezeigt werden, dass die radiologische Diagnostik der DRG B70B und B70D am Krankenhaus der Barmherzigen Brüder Trier mit einer positiven Erlös-Kosten-Relation erbracht wurde [7].

\section{Der ärztliche Zentrumsleiter („Chefarzt") und die Füh- rungspersonen müssen zu den Prozessanforderungen und den notwendigen Strukturen im DRG-Zeitalter passen.}

Prozessoptimierung beginnt in den Köpfen der Führungskräfte. Wenn der Chefarzt und die Führungskräfte nicht die Notwendigkeit erkannt haben, in Gesamtprozessen (Imaging Pathways) zu denken und zu optimieren, werden alle strukturellen und personellen Voraussetzungen nichts nutzen [8].

Der „ideale“ Abteilungs-/Zentrumsleiter soll eine außergewöhnlich hohe Fachkompetenz, eine herausragende Sozialkompetenz und ein großes Managementwissen besitzen. Doch derart vollkommene Eigenschaften sind im Personalmarkt selten zu bekommen. Personen müssen zu den notwendigen Prozessen und Strukturen passen und nicht umgekehrt. Die Leitung eines großen multidisziplinären und multiprofessionellen Zentrums im DRGZeitalter erfordert ein anderes Anforderungsprofil als die Leitung einer kleineren Fachabteilung. Während bei einer kleineren Abteilung die Qualität der persönlichen Leistungserbringung im Vordergrund steht, sind in großen Zentren Sozialkompetenz und Managementwissen herausragende Eigenschaften eines Leiters (Funktion des Dirigenten vor einem großen Orchester). Bei attraktiven Arbeitsbedingungen muss Expertenwissen in Teilbereichen im Markt „eingekauft" werden ( $\bullet$ Abb. 4).

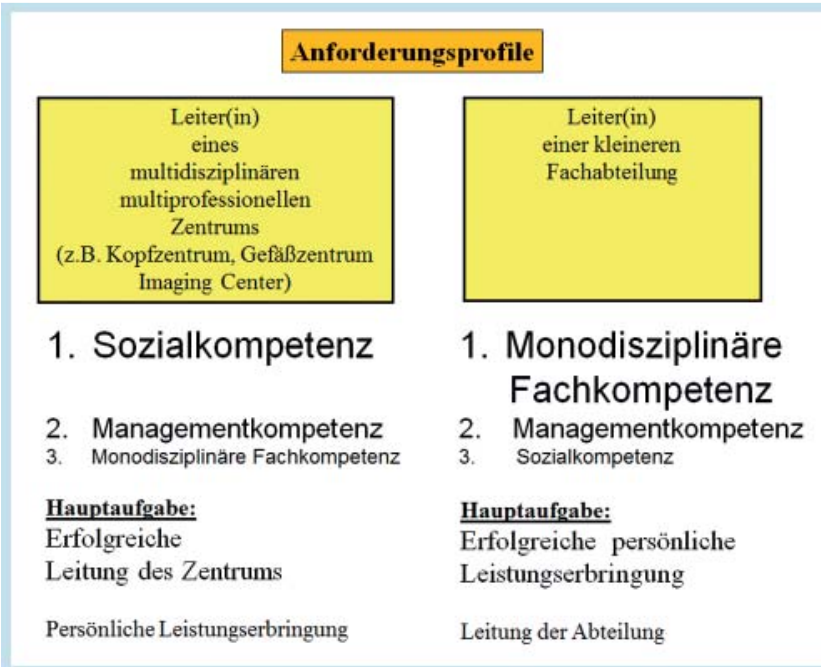

Abb.4 Anforderungsprofil an Leiter von Abteilungen und Zentren.

Moderne, größere Abteilungen und Zentren erfordern nicht einen „Chef der Ärzte“ sondern einen Leiter der Abteilung oder des Zentrums. In einem Zentrum ist in der Regel die Leitung und Führung von Ärzten verschiedener Disziplinen, von technischen Assistenten, Servicekräften und qualifizierten Verwaltungsmitarbeitern notwendig. Hilfreich ist dabei die Unterstützung durch eigene Stabsstellen, welche „hochkarätig“ qualifiziert (keine Auszubildenden, Berufsanfänger oder Studenten) besetzt sind. In dieser Expertenorganisation ist der Leiter umgeben von Mitarbeitern mit höherer Fachkompetenz in Teilbereichen der eigenen Fachdisziplin und zusätzlich von Experten anderer Fachdisziplinen (internes PEERSystem).

Insbesondere in großen Krankenhäusern und Universitätskliniken hat sich die Rolle des Abteilungsleiters vom „Gott in Weiß“ zum Steuermann eines komplexen Netzwerks von Experten entwickelt (Expertenorganisation). Primäre Aufgabe ist dabei eine Leistungserbringung der Abteilung im DRG-Zeitalter mit hoher 
Qualität und Wirtschaftlichkeit und die Schaffung einer positiven Arbeitsatmosphäre, um gute Mitarbeiter (Experten) zu halten und neue hoch qualifizierte Fachkräfte zu gewinnen [12]. Für einen Chefarzt kann es der größte persönliche Erfolg sein, die Abwanderung einer motivierten und hoch qualifizierten Führungskraft verhindert zu haben (Mitarbeiterbindung) oder die Klinikleitung von der Anschaffung eines zusätzlichen MRT mit Spitzentechnologie überzeugt zu haben. Auch das kostet viel Zeit und Energie.

\section{Das „Imaging Center“ (Zentrum für Radiologie, Neuroradiologie, Sonografie und Nuklearmedizin) im Krankenhaus der Barmherzigen Brüder Trier $\nabla$}

Am Beispiel des seit 7 Jahren bestehenden Imaging Centers im Krankenhaus der Barmherzigen Brüder Trier sollen Anregungen für die Einführung dieser neuen Organisationsform dargestellt werden.

Das Imaging Center im Krankenhaus der Barmherzigen Brüder Trier wird kooperativ von einem ärztlichen Zentrumsleiter und einer medizinisch technischen Zentrumsleiterin geführt. Beide sind dem Direktorium direkt verantwortlich und führen gemeinsam mit dem Direktorium Zielvereinbarungsgespräche für das Zentrum. Der Zentrumsleitung sind direkt eigene Stabsstellen EDV und Organisationsentwicklung/Prozessoptimierung mit entsprechend qualifizierten Fachkräften (Dipl. Ing. FH, Diplom-Ökonom) zugeordnet. Aufgabe der Stabsstellen ist eine weitgehend eigenverantwortliche Arbeit in den Bereichen EDV, Prozessoptimierung und Organisationsentwicklung. Sowohl zur Unterstützung der Zentrumsleitung und der Krankenhausverwaltung als bei der internen und externen Kommunikation erfüllen Stabsstellen wichtige Funktionen im Rahmen der täglichen Organisation und strategischen Weiterentwicklung des Zentrums ( $\bullet$ Abb.5).

Das Imaging Center gliedert sich in 5 Sektionen:

- $\mathrm{CT} / \mathrm{MRT}$

- Angiografie/Durchleuchtung/Projektionsradiografie

- Nuklearmedizin

- zentrale interdisziplinäre Sonografie

- Neuroradiologie

Die Sektionen werden fachlich und organisatorisch weitgehend eigenständig von Sektionsleitern geführt. Entsprechend der Vertragssituation sind dies ltd. Ärzte oder Oberärzte. Während der Zentrumsleitung die medizinische und ökonomische Gesamtverantwortung zugeordnet ist, sind die Sektionsleiter in ihren Teilbereichen in medizinischen und organisatorischen Entscheidungen eigenverantwortlich tätig.

Das Zentrum hat 69 Mitarbeiter, davon 25 Ärzte, 42 medizinisch technische Assistenten und Organisationskräfte, einen Ökonomen und einen EDV-Spezialisten. Mit einem Personal- und Sachmittelbudget von ca. 5,8 Mio. Euro wird ein Leistungsvolumen von ca. 161 Mio. Leistungspunkten (GOÄ) erreicht. Eigenständig, aber dem Zentrum organisatorisch angegliedert, ist ein MVZ für Radiologie und Nuklearmedizin des Krankenhauses der Barmherzigen Brüder.

Die für das Krankenhaus zentrale interdisziplinäre Sonografie (ZIS) stellt in Deutschland eine neue Organisationsform dar. In dieser Sektion arbeiten Ultraschall-Spezialisten (Internisten, Angiologen, Chirurgen, Radiologen, Nuklearmediziner) mit ausgewiesener Expertise und hochwertigsten Geräten. Die sonografische Ausbildung für die gesamte Klinik erfolgt in dieser Sektion. Sono-Bilder und -Sequenzen (KM-Sonografie) sind fester Bestandteil des

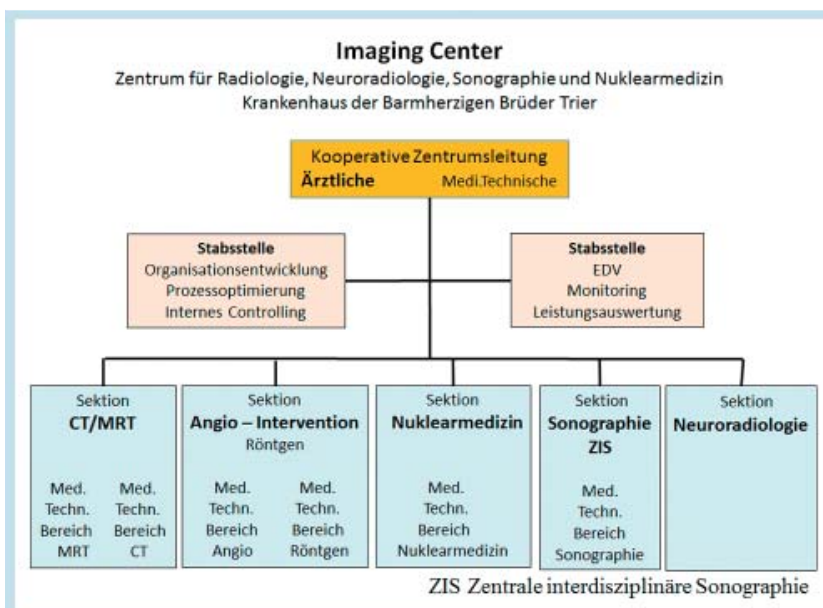

Abb. 5 Organigramm des Imaging Centers (Krankenhaus der Barmherzigen Brüder Trier).



Abb. 6 MRT-Utilization (mit freundlicher Unterstützung von Philips Health Management, A. van Est).

PACS-Systems und werden bei klinischen Konferenzen gleichberechtigt mit radiologischen Bildern von Experten demonstriert. Es besteht die Möglichkeit, insbesondere bei Interventionen zur „Online"-Fusion von Sono-Bildern mit MRT- und CT-Bildern. Der Sonografie kommt damit nicht nur der Stellenwert einer orientierenden Einstiegsdiagnostik vor einem gezielten Einsatz aufwendiger Methoden (z.B. CT, MRT, PET) zu. Die Ultraschalldiagnostik hat den Status einer Referenzmethode, die in vielen Fällen eine weitergehende bildgebende Diagnostik überflüssig macht. Zahlreiche Interventionen (z. B. Biopsien, Schmerzblockaden) werden zunehmend nicht mehr CT-, sondern Sono-gesteuert (Reduktion der Strahlendosis!) durchgeführt.

Radiologische Großgeräte müssen mit hoher Effizienz betrieben werden. Utilization Management ( $\boldsymbol{Q} \mathbf{A b b} . \mathbf{6})$ erfordert geeignete IT-Werkzeuge und ein Arbeitsklima, in dem mit den beteiligten Mitarbeitern offen und kritisch über das Thema Geräteauslastung gesprochen werden kann. Auch dieses Thema erfordert Übung und eine Zeit der Vertrauensbildung. Für einen hohen Auslastungsgrad bedeuten ungeplante Wartezeiten auf den Patienten durch eine nicht optimale Organisation der Patientenzuführung Leerlauf in der Gerätenutzung, der im laufenden Betrieb häufig nicht mehr 
aufzuholen ist. Im Imaging Center wurde aus diesem Grund in $\mathrm{Zu}-$ sammenarbeit mit der Pflegedienstleitung eine Clearingstelle eingerichtet. Kommt es durch organisatorische Probleme zu längeren Wartezeiten bei der Patientenzuführung von den Stationen, so wird dieses mit einem elektronischen „Ticket-System“ der Clearingstelle (Personen: Stabsstelle Radiologie/Pflegedienstleitung) gemeldet. Diese kümmert sich dann systematisch um die Ursachen, spricht mit den Prozessbeteiligten und versucht interprofessionelle Lösungen für die Zukunft zu finden. Eine statistische Betrachtung weist gegebenenfalls auf sich wiederholende Ursachen mit hohem Handlungsbedarf hin. Das Ergebnis der Clearingstelle ist eine deutliche Reduktion der Geräteleerlaufzeiten wegen fehlender Patientenzuführung [9].

In radiologischen Abteilungen im Krankenhaus ist ein professionelles Management mit einer effektiven Steuerung der Abläufe und einer klaren strategischen Ausrichtung auf der Basis von Kennzahlen Voraussetzung für eine erfolgreiche medizinische Tätigkeit.

Der gesamte Imaging-Prozess muss optimiert werden („Sie haben ein diagnostisches Problem - wir lösen dieses Problem in einem optimalen medizinischen und ökonomischen Gesamtprozess“). Dies setzt einen geeigneten internen Organisationsprozess mit eigenem Hol- und Bringdienst voraus. So wird der stationäre Patient direkt von der Station zum Imaging-Prozess z. B. erst zur Röntgenaufnahme, dann direkt zur Sonografie, dann direkt zur nuklearmedizinischen Untersuchung und anschließend wieder zur Station geführt. Diese zusätzliche interne Terminkoordination und Organisation ist keine leichte Aufgabe. Sie erfordert an allen Arbeitsplätzen ein kontinuierliches Monitoring (Organizer - Aktualisierungsintervall $1 \mathrm{Min}$ ). Wo ist gerade welcher Patient? Wie lange wartet der Patient schon vor Ort auf die Untersuchung? Welche weiteren Untersuchungen sind noch geplant? Gibt es eine Möglichkeit der direkten Überleitung?

Dem Imaging Center stehen kurzfristig (täglich), mittelfristig (wöchentlich/monatlich) und langfristig (jährlich) Kennzahlen zur Verfügung. Aufgabe der täglichen Kennzahlen ist das Monitoring der laufenden Aktivitäten, Aufgabe der wöchentlichen/ monatlichen Kennzahlen das Controlling der Datenqualität, der Prozessqualität und der Zielerreichung, Aufgabe der jährlichen Kennzahlen die Festlegung langfristiger Strategien (Marketing) und der Vergleich mit externen Leistungsanbietern (Benchmarking) ( $\triangle$ Abb. 7) [10]. In einer Jahreskonferenz werden die Ergebnisse des Zentrums im Vergleich zu den Zielen für alle Mitarbeiter transparent gemacht.

Als Beispiel für einen bestehenden Imaging Pathway soll der Prozess „Staging Rektum Karzinom“ im Krankenhaus der Barmherzigen Brüder Trier dargestellt werden. Dieser Imaging Pfad beginnt mit der entsprechenden klinisch-diagnostischen Aufgabenstellung („Staging“) an das Imaging Center und endet mit einer Zusammenfassung der einzelnen Befunde zu einer Gesamtbeurteilung.

Vor der Einführung des Pathway „Staging Rektum Karzinom“ wurden sämtliche Untersuchungen (Computertomografie Abdomen, Becken, Thorax - MRT Becken - Endosonografie - Sonografie Abdomen, Becken, evtl. Lungenaufnahme) parallel jeweils mit höchster Qualität durchgeführt. Der Weg, einen gemeinsamen Imaging Pathway zu definieren und zu organisieren, war schwierig und zeitaufwendig. Insbesondere in der Einführungsphase kann ein konsequentes Monitoring mit einem geeigneten Informationsmanagement nur „als Chefsache“ den Imaging-Pfad „am Leben“ halten. Nach einiger Zeit muss dann jedoch eine retrospektive Studie den medizinischen und ökonomischen Erfolg nachweisen (๑ Abb.8).

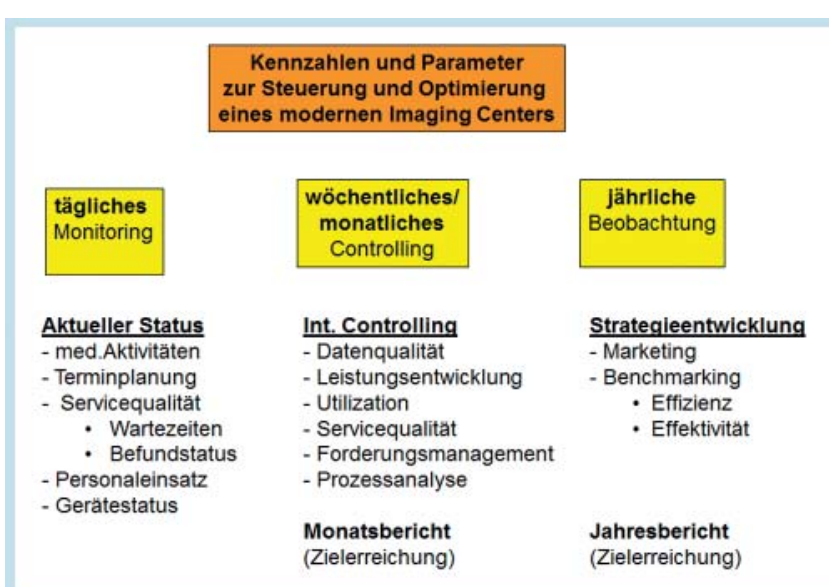

Abb. 7 Kennzahlen zur Steuerung und Optimierung eines modernen Imaging Centers.

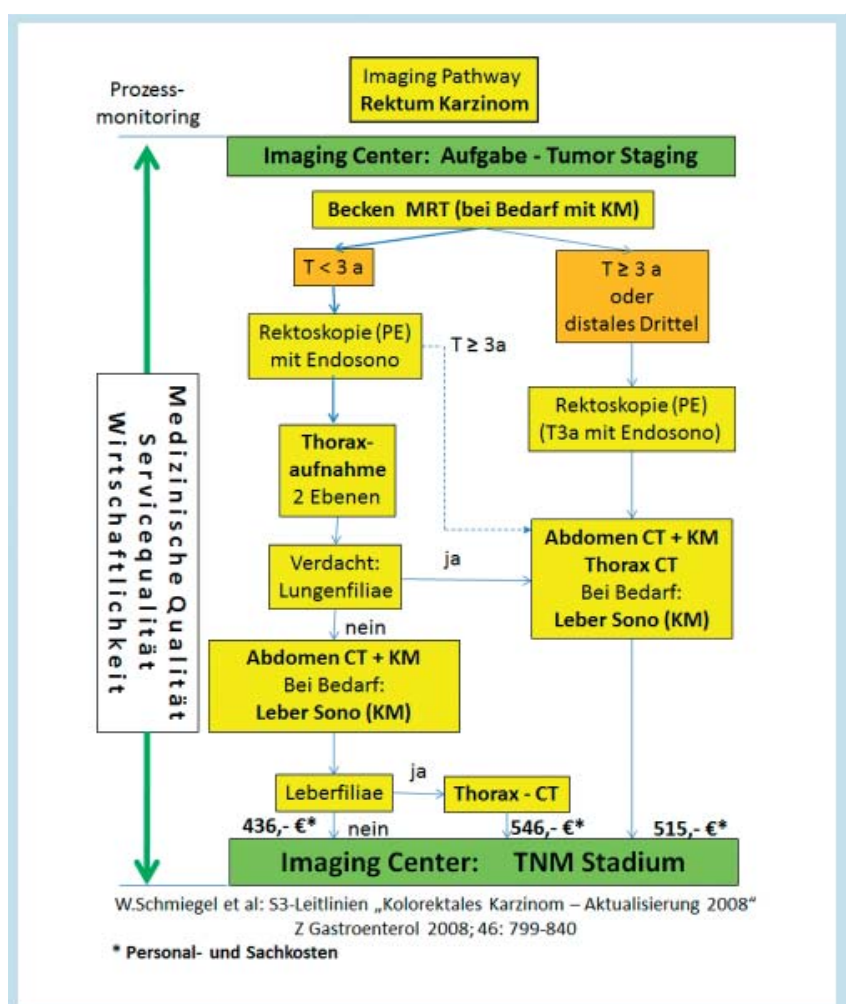

Abb. 8 Imaging Pathway: Staging Rektum Karzinom.

Das Monitoring und die Optimierung des Imaging Pathways stellen hohe Anforderungen an die Qualität der Abläufe im Imaging Center. Die Untersuchungen werden vom Imaging Center ausgewählt und durchgeführt entsprechend dem mit den klinischen Partnern verabredeten Imaging Pathway - am Anfang steht die MRT-Untersuchung! Die Ablauforganisation erfordert teilweise eine direkte klinische Entscheidung (Weichenstellung) mit einer direkten Überleitung des Patienten zu einer weiteren Untersuchung im Imaging Center und eine taggleiche Befundung (Spracherkennungssysteme). Zum Beispiel ordnet bei einem fraglichen CT-Befund der Leber der Radiologe direkt die Sonografie an (eine neue Dimension: Anforderungen innerhalb des Imaging Centers passen nicht zur alten Verrechnung von 
Einzelleistungen mit den Zuweisern). Der Patient wird direkt in die Sonografie-Abteilung weitergeleitet und umgehend untersucht. Der Radiologe kann das Ergebnis in seinen CT-Befund einfließen lassen. Direkt nach Festlegung des lokalen Stadiums im MRT erfolgt die Entscheidung, ob die „prophylaktisch“ angemeldete Endosonografie durchgeführt werden muss. Am Ende des Imaging Pathways steht der Gesamtbefund des Imaging Centers (eine Fragestellung - ein Termin - eine Antwort).

\section{Aktuelle Ergänzung}

Inzwischen wurde ein internes Bereichskontrolling (CT, MRT, Sono ...) mit systematischem Berichtswesen im Zentrum etabliert.

Es wurde mit regelmäßigen klinischen Konferenzen zum Thema „Imaging Pathways - Patientenbelastung und Ökonomie“ begonnen.

Eine Analyse durch eine externe Unternehmensberatung ergab eine Rechtfertigung des ärztlichen Stellenplanes und eine Bestätigung der Refinanzierung des ärztlichen Dienstes im Zentrum durch die entsprechenden DRG-Anteile.

\section{Literatur}

1 Busch HP. Managementhandbuch für Radiologen. Thieme Verlag; 2008

2 Busch HP. Managementhandbuch für Chefärzte. Thieme Verlag; 2012

3 Busch HP. Wirtschaftlichkeitsreserven im Krankenhaus - Eine kritische Analyse der Prozesse, Strukturen und Personen. Arzt und Krankenhaus 2012; 6: 176-179
4 Busch HP. In Prozessen denken und optimieren - eine Herausforderung für Krankenhäuser. Das Krankenhaus 2011; 5: 459-464

5 Busch HP. Prozessoptimierung im Krankenhaus - Behandlungsabläufe im Überangebot bildgebender Untersuchungsmethoden. Arzt und Krankenhaus 2012; 2: 46-51

6 Busch HP. Benchmarking of Radiologic Departments - Starting point for successful process optimization. Fortschr Röntgenstr 2010; 182: $221-228$

7 HopfJ. Bildgebende Verfahren („Imaging“) bei der Akutbehandlung des ischämischen Schlaganfalls - Eine retrospektive Analyse aus medizinischer und ökonomischer Sicht. Dissertation an der Medizinischen Fakultät Mannheim der Ruprecht-Karls-Universität Heidelberg 2012

8 Busch HP. Das Berufsbild „Chefarzt“ im Wandel. Das Krankenhaus 2011; 10: $312-315$

9 Frewer W, Schuh H, Busch HP. Schnittstellenmanagement im Rahmen kontinuierlicher Prozessoptimierung von klinischen Behandlungspfaden. Das Krankenhaus 2012; 10: 1003 -1009

10 Busch HP. Kennzahlen und Informationen zur Steuerung und Optimierung radiologischer Abteilungen (Imaging Center). Radiologie up2date 2011; 3: $267-282$

11 Schmiegel W et al. S3-Leitlinien „Kolorektales Karzinom“ - Aktualisierung. Z Gastroenterol 2008; 46: 799-840

12 Beitzel KI, Ertl L, Grosse C et al. Job satisfaction of radiologists in Germany - status quo. FortschrRöntgenstr 2011; 183: 749-757

13 Hoffstetter P, Hammer S, Rohrl S et al. As Time Goes By - Is it Worth Intensifying Patient Care During the Waiting Period for Radiological Examinations? Fortschr Röntgenstr 2012, eFirst 31.7.2012

14 Lorenzen J, Habermann C, Utler C et al. Certified quality management according to DIN ISO 9001 in a radiology department at a university hospital: measurable changes in academic quality indicators? Fortschr Röntgenstr 2009; 181: 970-978

15 Teichgraber $U$, de Bucourt M. Qualitätsmanagementsysteme in der Radiologie: Umsetzung in der Klinik und Praxis. Fortschr Röntgenstr 2010; 182: 986-992

16 Frewer W, Busch HP. Financial Management in the imaging department. Imaging Management 2012; 12: 20-23

\section{Buchbesprechung}

Operative und interventionelle Gefäßmedizin

Debus ES, Gross-Fengels W

1. Aufl., Springer: Berlin, 993 S., 855 Abb. in Farbe, $229 €$ als Hardcover, ISBN: 978-3-642-01708-7

Das vorliegende Buch richtet sich als klassisches Lehrbuch und Nachschlagewerk an alle vaskulär tätigen Ärzte.

Die beiden Herausgeber und ein interdisziplinäres Team von 80 Mitautoren bündeln in diesem Buch ihr Expertenwissen und schaffen so ein Werk, welches das Gesamtgebiet der vaskulären Medizin umfassend und detailliert abdeckt.

Im ersten Teil werden grundlegende Themen wie Anatomie, Physiologie und Pathophysiologie des Gefäßsystems abgehandelt. Hervorzuheben sind hierbei die sehr gut aufgearbeiteten anatomischen Tafeln und die detaillierten Angaben zum operativen Zugangsweg. Die pathophysiologischen Zusammenhänge werden durch zahlreiche, eingängige Schemata und Tabellen qualitativ anspruchsvoll dargestellt. Ausführlich werden in diesem Teil auch die vaskulären Untersuchungsmethoden, Indikationsstellung, chirurgische und interventionelle Techniken, sowie die Materialkunde, erläutert. Im zweiten und dritten Teil werden die regionalen arteriellen bzw. venösen Erkrankungen, im vierten und letzten Teil regionenübergreifende Erkrankungen der Arterien, Venen und Lymphgefäße abgehandelt. Die einzelnen Kapitel folgen einem einheitlichen, strukturierten Schema. Dabei wird bei den einzelnen Krankheitsbildern auf Definiton, Epidemiologie, Pathogenese, Klinik sowie Diagnostik eingegangen. Schließlich werden interventionelle bzw. operative Therapieoptionen durch hervorragendes Bildmaterial verdeutlicht. Ebenso wird ausführlich auf das prä- und postoperative Management eingegangen. Die einzelnen Kapitel werden durch Ausführungen zur aktuellen Studienlage abgerundet. Tabellen und farblich hervorgehobene Merksätze steuern zur Übersichtlichkeit der einzelnen Kapitel bei.

Fazit:

Das vorliegende Buch ist ein didaktisch hervorragend konzipiertes, klar strukturiertes Lehrbuch.

Besonders hervorzuheben sind die übersichtliche Gliederung und Systematik der einzelnen Kapitel. Neben grundlegenden Themen stellt dieses Buch umfassend die aktuellen Therapiekonzepte aller Gefäßerkrankungen dar und ist so als Referenzwerk für jeden vaskulär tätigen Arzt unbedingt zu empfehlen.

Dr. Andreas Müller, Bonn 